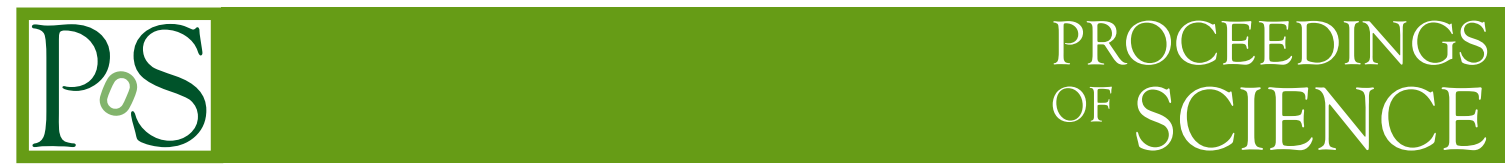

\title{
Hard diffractive scattering from soft color screening effects
}

\author{
Roman Pasechnik*, Rikard Enberg, and Gunnar Ingelman \\ Department of Physics and Astronomy, Uppsala University, Box 516, SE-751 20 Uppsala, \\ Sweden \\ E-mail: Roman.Pasechnikefysast.uu.se
}

\begin{abstract}
We construct a QCD-based model where soft gluon rescattering between final state partons in deep inelastic scattering leads to events with large rapidity gaps and a leading proton. The model successfully describes the precise HERA data on the diffractive deep inelastic structure function in the whole available kinematical range.
\end{abstract}

35th International Conference of High Energy Physics

July 22-28, 2010

Paris, France

${ }^{*}$ Speaker. 


\section{Introduction}

Diffractive processes are sensitive to details of nonperturbative QCD dynamics and provide a way to probe the soft and semihard regimes directly. Diffractive events are characterized by a leading "target" particle, carrying most of the beam momentum, and a well separated produced hadronic system. The "gap" in between is connected to the soft part of the event and therefore to nonperturbative effects at a long space-time scale. Diffractive deep inelastic scattering (DDIS) offers a particularly good opportunity to explore the interplay between hard and soft physics due to precise data from HERA [ [ []].

To understand better this nonperturbative dynamics, we discuss here a QCD-based mechanism for soft gluon rescattering of final state partons. This mechanism leads to effective color singlet exchange and thereby to diffractive scattering. Our model was initially introduced in a recent letter [3], and was presented in Ref. [⿴囗木 in more detail. Here we give the essence of this new model and discuss its potential.

\section{Diffractive structure function}

Let us consider first the simplest case of the $q \bar{q}$ contribution shown in Fig. (I (left), which is the leading one for small invariant mass $M_{X}$ of system $X$.
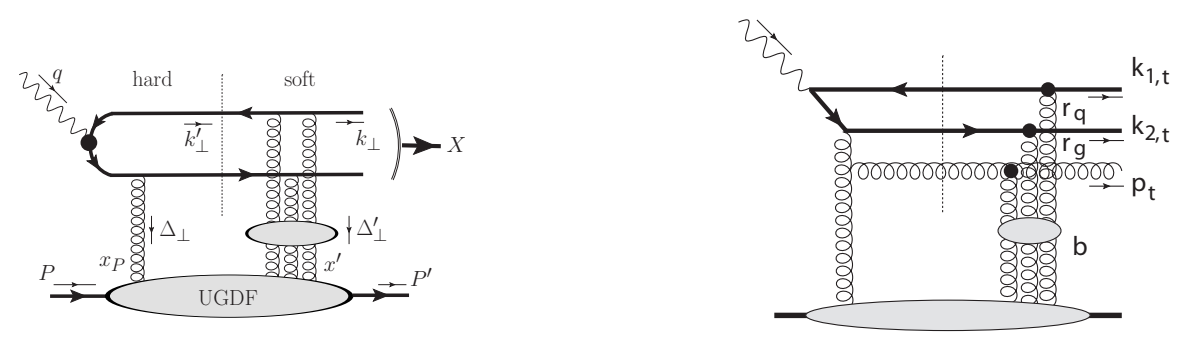

Figure 1: Amplitude of the process $\gamma^{*} p \rightarrow X p$ with $q \bar{q}$ dipole scattering contribution with all-order resummed soft gluon exchange (left) and gluon emission (right) giving a non-negligible contribution for large $M_{X}(\operatorname{small} \beta)$.

In impact parameter space, with $\mathbf{b}$ conjugate to $\Delta_{\perp}$, the total amplitude for the $\gamma^{*} p \rightarrow X p$ process can be written as a convolution of the hard scattering amplitude $\gamma^{*} g \rightarrow q \bar{q}$, the soft-gluon rescattering amplitude containing information about (almost) real quark/antiquark rescattering off the proton remnant, and the unintegrated gluon density describing coupling of the gluons to the proton target, i.e.

$$
M(\delta) \sim \int d^{2} b e^{-i \boldsymbol{\delta} \mathbf{b}} \hat{M}^{\text {hard }} \hat{M}^{\text {soft } \mathscr{V}} .
$$

where $\delta \equiv \sqrt{-t}=\left|\Delta_{\perp}+\Delta_{\perp}^{\prime}\right|$. The function $\mathscr{V}$ is the Fourier transform of the unintegrated gluon distribution function (UGDF), and will be specified below. This QCD factorization is schematically illustrated in Fig. W, and provided by the hard scale $\mu_{F}^{2}$ being the hardest quark virtuality $\mu_{F}^{2} \equiv$ $\varepsilon^{2}+k_{\perp}^{2}=z(1-z)\left(M_{X}^{2}+Q^{2}\right)$, where $\varepsilon=\sqrt{z(1-z)} Q$ and $k_{\perp}=\sqrt{z(1-z)} M_{X}$ are the energy and transverse momentum of a quark carrying the longitudinal momentum fraction $z$. 
The Fourier-transformed amplitudes $\hat{M}^{\text {hard }}$ and $\hat{M}^{\text {soft }}$ lead to the longitudinal and transverse contributions to the diffractive structure functions $F_{L, T}^{D,(4)}\left(x_{P}, Q^{2}, \beta, t\right)$ :

$$
\begin{aligned}
& x_{P} F_{L}^{D(4)}=\mathscr{S} Q^{4} M_{X}^{2} \int_{z_{\text {min }}}^{\frac{1}{2}} d z(1-2 z) z^{2}(1-z)^{2}\left|J_{L}\right|^{2} \\
& x_{P} F_{T}^{D(4)}=2 \mathscr{S} Q^{4} \int_{z_{\text {min }}}^{\frac{1}{2}} d z(1-2 z)\left\{(1-z)^{2}+z^{2}\right\}\left|J_{T}\right|^{2},
\end{aligned}
$$

where $\mathscr{S}=\sum_{q} e_{q}^{2} /\left(2 \pi^{2} N_{c}^{3}\right)$ sums over light quark charges $e_{q}$, and

$$
\begin{aligned}
& J_{L}=i \alpha_{s}\left(\mu_{F}^{2}\right) \int d^{2} \mathbf{r} d^{2} \mathbf{b} e^{-i \boldsymbol{\delta} \mathbf{b}} e^{-i \mathbf{r} \mathbf{k}_{\perp}} K_{0}(\varepsilon r) \mathscr{V}(\mathbf{b}, \mathbf{r})\left[1-e^{\mathscr{A} \mathscr{W}}\right] \\
& J_{T}=i \alpha_{s}\left(\mu_{F}^{2}\right) \int d^{2} \mathbf{r} d^{2} \mathbf{b} e^{-i \boldsymbol{\delta} \mathbf{b}} e^{-i \mathbf{r k}_{\perp}} \varepsilon K_{1}(\varepsilon r) \frac{r_{x} \pm i r_{y}}{r} \mathscr{V}(\mathbf{b}, \mathbf{r})\left[1-e^{\mathscr{A} \mathscr{W}}\right] .
\end{aligned}
$$

The expression in square brackets represents the result of exponentiation of soft gluon final-state interactions in the large $N_{c}$ limit, with $\mathscr{A}=2 \pi i C_{F} \alpha_{s}\left(\mu_{\text {soft }}^{2}\right)$ and $\mathscr{W}=(1 / 2 \pi) \ln (|\mathbf{b}-\mathbf{r}| /|\mathbf{b}|), K_{0,1}$ are the Bessel functions, and

$$
\mathscr{V}(\mathbf{b}, \mathbf{r})=\frac{1}{\alpha_{s}\left(\mu_{\mathrm{soft}}^{2}\right)} \int \frac{d^{2} \Delta_{\perp}}{(2 \pi)^{2}} \sqrt{x_{P}} \mathscr{F}_{g}^{\mathrm{off}}\left(x_{P}, \Delta_{\perp}^{2}\right)\left\{e^{-i \mathbf{r} \boldsymbol{\Delta}_{\perp}}-e^{i \mathbf{r} \boldsymbol{\Delta}_{\perp}}\right\} e^{i \mathbf{b} \boldsymbol{\Delta}_{\perp}} .
$$

Here, $\mathscr{F}_{g}^{\text {off }}\left(x_{P}, \Delta_{\perp}^{2}\right)$ is the generalized unintegrated gluon distribution function (UGDF), which can be related with the integrated gluon PDF $x_{P} g\left(x_{P}, \mu_{F}^{2}\right)$, and $\mu_{\text {soft }} \ll \mu_{F}$ is the typical soft scale of the process given by the gluon virtuality $\sim \Delta_{\perp}^{2}$. The QCD coupling at this scale $\alpha_{s}\left(\mu_{\text {soft }}\right)$ is calculated in the framework of the singularity-free Analytic Perturbation Theory and fixed to be $\sim 0.7$ at $\mu_{\text {soft }} \sim \Lambda_{Q C D}$

At large- $M_{X}$, the gluon emission may be important. In principle, gluons may be radiated from both the $q \bar{q}$ dipole and the hard gluon. The gluons emitted from the quarks are dominantly soft and move collinearly with the quarks, and do not significantly change the invariant mass of the final system $X$. Large $M_{X}$ is instead obtained by gluon emission with longitudinal momentum fraction $z^{\prime}$ from the hard gluon, as illustrated in Fig. W(right), and is realized when $z^{\prime} \ll z$, such that $M_{X}^{2} \simeq M_{q \bar{q}}^{2}+p_{\perp}^{2} / z^{\prime} \gg M_{q \bar{q}}^{2}, M_{q \bar{q}}^{2} \simeq\left(k_{\perp}^{2}+m_{q}^{2}\right) / z(1-z)$.

Also, due to momentum conservation, the maximal $M_{X}$ at fixed $z^{\prime}$ occurs in the limit $p_{\perp} \sim$ $k_{1, \perp} \gg k_{2, \perp}$, which corresponds to $r_{1} \ll r_{2}$ in impact parameter space. It turns out that the $q \bar{q} g$ and $q \bar{q}$ dipole contributions in this limit saturate to the same value, i.e. $M_{q \bar{q} g}^{\text {soft }} \simeq M_{q \bar{q}}^{\text {soft }}$ at large invariant masses $M_{X}$. In particular, this means that the scattering of the $q \bar{q} g$ system off the proton for large $M_{X}$ can not be reduced to the scattering of the gluonic dipole. For more details, see Ref. [䧃].

\section{Numerical results}

The HERA data [ [ [ ] $]$ on DDIS are given in the form of the reduced cross section $x_{P} \sigma_{r}^{D(3)}$ expressed in terms of the diffractive structure functions $F_{L, T}^{D(3)}\left(x_{P}, Q^{2}, \beta\right)$. The momentum transfer $t$ is integrated over since in most of the data the leading proton is not observed, and diffraction is equivalently defined through a large rapidity gap. In Fig. $\square$ we compare the latest ZEUS data [四] with the numerical evaluation of our model. A generally very good agreement is found with CTEQ6L1 gluon PDF [ [ل]], except for very small values of $Q$ and $M_{X}$, where the QCD factorization scale $\mu_{F}$ becomes very small. 


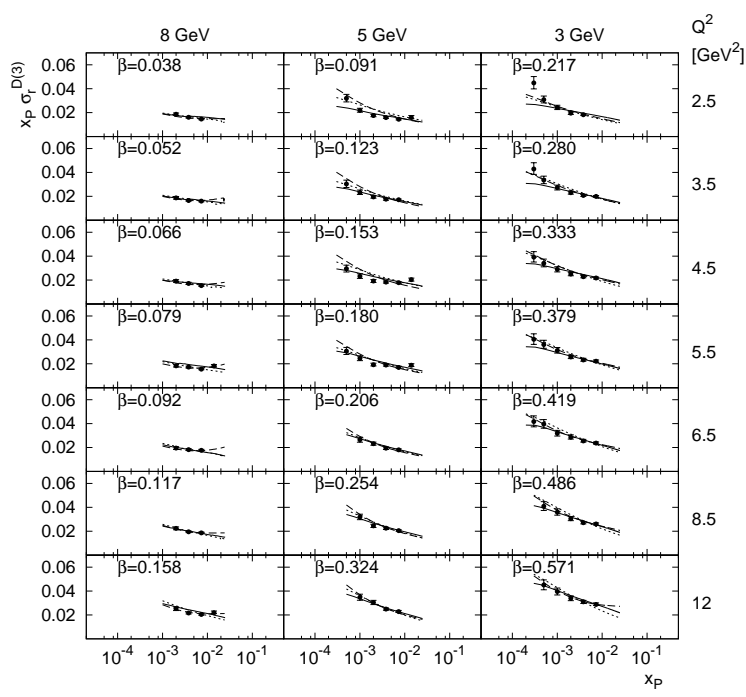

Figure 2: The reduced cross section $x_{P} \sigma_{r}^{D(3)}\left(x_{P}, \beta, Q^{2}\right)$ for $M_{X} \leq 3,5,8 \mathrm{GeV}$ and $Q^{2}<12 \mathrm{GeV}^{2}$, where the lowest $x_{P}$-values are reached. ZEUS data [四] are compared to the model results using different extrapolations of the gluonic density at small $x_{P}$ and $\mu_{F}^{2}$ as detailed in Ref. [四].

\section{Conclusion}

We have developed a proper QCD framework for diffractive hard scattering, which contains both hard and soft dynamics. The hard part produces a well-defined state of emerging partons, and the soft part is the rescattering of these partons with the color field of the proton remnant.

Numerical evaluation of the analytical results gives good agreement with the precise HERA data on the diffractive deep inelastic cross section. The $q \bar{q}$ contribution is indeed dominant, but at $\beta \lesssim 0.2$, the $q \bar{q} g$ contribution is important. While CTEQ6L1 works well at large $\mu_{F}$ scales and large $x_{P}$, at very small $x_{P} \lesssim 5 \times 10^{-4}$ and scales $\mu_{F}^{2} \sim 1 \mathrm{GeV}^{2}$ the gluon density in the proton is very poorly known and gives a complication in the comparison with the few HERA data points in this extreme region.

The new model discussed here has the potential to be applied in many different exclusive and diffractive processes. Having demonstrated that our theoretical formalism for DDIS does describe HERA data, one may then extract the part describing the multigluon exchange process and apply it to other hard processes, including $p \bar{p}$ collisions at Tevatron and $p p$ collisions at LHC.

\section{References}

[1] S. Chekanov et al. [ZEUS Collaboration], Nucl. Phys. B 816, 1 (2009).

[2] M. Derrick et al., Phys. Lett. B 315, 481 (1993); Phys. Lett. B 346, 399 (1995); T. Ahmed et al., Nucl. Phys. B 429, 477 (1994); Nucl. Phys. B 435, 3 (1995).

[3] R. Pasechnik, R. Enberg and G. Ingelman, Phys. Lett. B (in press), arXiv:1004.2912 [hep-ph].

[4] R. Pasechnik, R. Enberg and G. Ingelman, Phys. Rev. D82, 054036 (2010).

[5] J. Pumplin et al., JHEP 0207, 012 (2002). 